2. Puente XS, Pinyol M, Quesada V, Conde L, Ordonez GR, Villamor $\mathrm{N}$, et al. Whole-genome sequencing identifies recurrent mutations in chronic lymphocytic leukaemia. Nature. 2011;475:101-5.

3. Rosati E, Baldoni S, De Falco F, Del Papa B, Dorillo E, Rompietti $\mathrm{C}$, et al. NOTCH1 aberrations in chronic lymphocytic leukemia. Front Oncol. 2018;8:229.

4. Arruga F, Gizdic B, Bologna C, Cignetto S, Buonincontri R, Serra $\mathrm{S}$, et al. Mutations in NOTCH1 PEST domain orchestrate CCL19driven homing of chronic lymphocytic leukemia cells by modulating the tumor suppressor gene DUSP22. Leukemia. 2017;31:1882-93.

5. De Falco F, Sabatini R, Falzetti F, Di Ianni M, Sportoletti P, Baldoni S, et al. Constitutive phosphorylation of the active Notch1 intracellular domain in chronic lymphocytic leukemia cells with NOTCH1 mutation. Leukemia. 2015;29:994-8.

6. Pozzo F, Bittolo T, Arruga F, Bulian P, Macor P, Tissino E, et al. NOTCH1 mutations associate with low CD20 level in chronic lymphocytic leukemia: evidence for a NOTCH1 mutation-driven epigenetic dysregulation. Leukemia. 2016;30:182-9.

7. Quijada-Alamo M, Hernandez-Sanchez M, Robledo C, Hernandez-Sanchez JM, Benito R, Montano A, et al. Nextgeneration sequencing and FISH studies reveal the appearance of gene mutations and chromosomal abnormalities in hematopoietic progenitors in chronic lymphocytic leukemia. J Hematol Oncol. 2017;10:83.
8. Kikushige $\mathrm{Y}$, Ishikawa $\mathrm{F}$, Miyamoto $\mathrm{T}$, Shima $\mathrm{T}$, Urata $\mathrm{S}$, Yoshimoto G, et al. Self-renewing hematopoietic stem cell is the primary target in pathogenesis of human chronic lymphocytic leukemia. Cancer Cell. 2011;20:246-59.

9. Fabbri G, Holmes AB, Viganotti M, Scuoppo C, Belver L, Herranz $\mathrm{D}$, et al. Common nonmutational NOTCH1 activation in chronic lymphocytic leukemia. Proc Natl Acad Sci USA. 2017;114:E2911-E2919.

10. Di Ianni M, Baldoni S, Del Papa B, Aureli P, Dorillo E, De Falco $\mathrm{F}$, et al. NOTCH1 is aberrantly activated in chronic lymphocytic leukemia hematopoietic stem cells. Front Oncol. 2018;8:105.

11. Bray SJ. Notch signalling in context. Nat Rev Mol Cell Biol. 2016;17:722-35.

12. Lai AY, Wade PA. Cancer biology and NuRD: a multifaceted chromatin remodelling complex. Nat Rev Cancer. 2011;11:588-96.

13. Yatim A, Benne C, Sobhian B, Laurent-Chabalier S, Deas O, Judde JG, et al. NOTCH1 nuclear interactome reveals key regulators of its transcriptional activity and oncogenic function. Mol Cell. 2012;48:445-58.

14. Yan XJ, Dozmorov I, Li W, Yancopoulos S, Sison C, Centola M, et al. Identification of outcome-correlated cytokine clusters in chronic lymphocytic leukemia. Blood. 2011;118:5201-10.

15. Ghia P, Strola G, Granziero L, Geuna M, Guida G, Sallusto F, et al. Chronic lymphocytic leukemia B cells are endowed with the capacity to attract $\mathrm{CD} 4^{+}, \mathrm{CD} 40 \mathrm{~L}^{+} \mathrm{T}$ cells by producing CCL22. Eur J Immunol. 2002;32:1403-13.

Leukemia (2019) 33:2956-2962

https://doi.org/10.1038/s41375-019-0523-8

Acute myeloid leukemia

\title{
TP53 abnormalities and chromosomal aneuploidy in acute panmyelosis with myelofibrosis
}

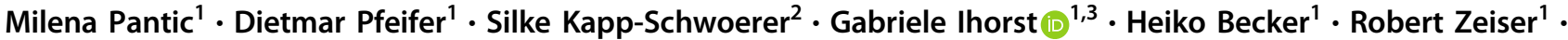 \\ Justus Duyster ${ }^{1} \cdot$ Annette Schmitt-Graeff $^{4}$
}

Received: 8 January 2019 / Revised: 16 April 2019 / Accepted: 9 May 2019 / Published online: 26 July 2019

(c) The Author(s) 2019. This article is published with open access

These authors contributed equally: Milena Pantic, Dietmar Pfeifer

Supplementary information The online version of this article (https:// doi.org/10.1038/s41375-019-0523-8) contains supplementary material, which is available to authorized users.

Annette Schmitt-Graeff

annette.schmitt-graeff@uniklinik-freiburg.de

1 Department of Hematology, Oncology and Stem Cell Transplantation, Medical Center - University of Freiburg, Faculty of Medicine, University of Freiburg, Germany, Freiburg, Germany

2 Department of Internal Medicine III, University Hospital of Ulm, Ulm, Germany

3 Clinical Trials Unit, Medical Center - University of Freiburg, Faculty of Medicine, University of Freiburg, Freiburg, Germany

4 University of Freiburg, Freiburg, Germany

\section{To the Editor:}

The World Health Organization (WHO) classification of acute myeloid leukemia (AML) has incorporated molecular genetic and cytogenetic aberrations in the definition of most entities [1]. The diagnosis of acute panmyelosis with myelofibrosis (APMF) is still not based on genomic changes but on clinicopathologic features and the exclusion of other myeloid malignancies, in particular AML with myelodysplasia-related changes (AML-MRC) [2]. APMF is a rapidly progressive hyperfibrotic subtype of "AML not otherwise specified" (AML NOS) and accounts for $<1 \%$ of AML cases [3]. This aggressive disease is characterized by rapid onset of cytopenia and constitutional symptoms in the absence of splenomegaly, previous history of myeloproliferative neoplasm (MPN) or myelodysplastic 
syndrome (MDS), and exposure to radiation or cytotoxic drugs. Blood smears are devoid of tear-drop-shaped cells but may contain dysplastic platelets and myeloid cells including rare blasts $[2,3]$. Histopathologic evaluation of core bone marrow (BM) biopsies is mandatory to recognize the hallmark of this myeloid neoplasm: "panmyelosis", i.e. trilineage myeloid proliferation with dysplastic erythropoiesis, abundant atypical megakaryocytes, and overt BM fibrosis $\geq$ grade 2 on a $0-3$ scale and $\geq 20 \%$ predominantly CD34-positive precursors/blasts often arranged in clusters (Fig. 1a-d) [3-5]. As a consequence of dry tap BM aspiration and rare circulating CD34-positive precursors/ blasts, genetic data are reported in a limited number of patients. An abnormal karyotype lacking recurrent aberrations was identified in most APMF cases with available cytogenetic information. Published cytogenetic data were similar to AML-MRC including aberrations of chromosome 5q, 7q/7, 8, and 11q [3, 4]. Two other studies detected different chromosomal aberrations involving chromosomes $3 q, 5,12,13 q$, and 22 [6, 7]. In a single APMF patient, an amplification of EVI1/MECOM within a derivative chromosome 8 was described [8]. In another study of four APMF patients no JAK2 V617F, MPL, or CALR mutations were detected [9].

The first step of our study was to identify in our files de novo AML patients who presented with relevant fibrosis at initial diagnosis. Among those, we retrospectively reevaluated cases with available BM biopsies for the presence of clinicopathologic features consistent with the 2016 WHO criteria for APMF. Cases with a previously proven MDS-type cytogenetic profile and prominent dysplasia involving $\geq 50 \%$ at least in two lineages as mandatory for AML-MRC were excluded. In addition, no cases with megakaryocytic abnormalities, such as arrangement in dense clusters characteristic for primary myelofibrosis (PMF) were considered. By consequently applying WHO criteria, we retrieved a cohort of 16 patients that we could assign to the APMF category (Fig. 1a-d, Table 1, Supplementary Table 1). Briefly, the core biopsies showed BM fibrosis grade 2-3 associated with a generally rather low frequency of CD34-positive myeloblasts (median 30\% in the total cohort), a proliferation of predominantly small dysplastic CD61-positive megakaryocytes and a macroblastic glycophorin C-positive and CD71-positive erythropoiesis with increased proerythroblasts. Using routine methods suitable for formalin-fixed paraffin-embedded (FFPE) material MPL W515L was absent in $5 / 5$ cases, while $1 / 5$ cases harbored a $J A K 2$ V617F mutation with a low $(5 \%)$ mutant allele burden. Karyotyping by metaphase analysis or FISH had been unsuccessful due to BM dry tap and low circulating CD34+ cells. Thus, we performed molecular studies on genomic DNA extracted from the diagnostic FFPE and EDTA-decalcified BM trephine biopsies by applying the OncoScanTM FFPE assay (Thermo Fisher Scientific, MA, USA) for whole genome tumor profiling [10]. Using stringent settings for gains and losses (Supplementary Materials and Methods) genomic copy number abnormalities (CNAs) were discovered in 94\% (15/16) of APMF patients. Based on the number of CNAs we could distinguish two groups within our APMF cohort. Six patients harbored $\leq 3$ (mean 2) CNAs and were referred to as low genomic complexity group (Fig. 1e, f, Table 1, Supplementary Table 2). The low complexity group showed mostly single but heterogeneous CNAs (Table 1). In contrast, 10 patients were assigned to a high genomic complexity group since $>3$ (mean 15) CNAs were identified. As a part of a complex genomic profile, the most frequent CNAs were losses of $17 \mathrm{p}, 5 \mathrm{q}$, and $7 \mathrm{q}$ (in 10, 9 , and 9 patients, respectively). Other MDS-related or MPNrelated CNAs occurred with lower frequency such as loss of $18 p$ in five patients and losses of $17 q, 18 q, 11 p$, and $3 p$, as well as a gain of $3 q$ in four patients, respectively (Fig. 1e, Table 1, Supplementary Table 2). The gains of chromosome $3 q, 8,12 q, 17 q$, and $21 q$, as well as loss of $7 q$ were the CNAs common to both groups and have been previously described in APMF [3, 6, 8].

DNA for mutational analysis (Supplementary Information) was only available in 5/16 patients (Table 1 ). Three of these patients were assigned to the high complexity group while two patients were included in the low complexity group. Molecular profiling revealed TP53 mutations in all of these five patients. Taken together with the copy number results, 12/16 patients harbored TP53 abnormalities (i.e. loss of $17 \mathrm{p}$ and/or TP53 mutation) including 3 with inactivation of both TP53 alleles (Table 1).

Further mutations were detected in the epigenetic regulators DNMT3A and TET2 (in 2/5 samples), signaling molecule $C B L$ (1/5 sample), and co-repressor $B C O R$ (in 2/5 samples). Four patients did not show TP53 abnormalities in the OncoScan assay but were not evaluable for TP53 mutation analysis. Thus, they were considered as "TP53 unknown".

As compared with other AML entities, the genetic alterations underlying APMF are not well characterized. An interesting finding that has not yet been described is the presence of TP53 aberrations in the large majority of APMF patients. In accordance, we frequently observed high levels of genomic complexity suggesting a high genomic instability universally associated with an adverse outcome in AML [11]. Patients of the large high complexity group (10/16 patients) exhibited a genetic profile overlapping with AML MRC. TP53 alterations are rare in de novo AML (about $8-14 \%$ ) but are closely associated with the presence of a complex karyotype and/or monosomal karyotype $[11,12]$. In a recent study, AML with TP53 mutations and chromosomal aneuploidies, such as deletions and monosomies of chromosomes 5, 7, 12, and 17, 
a

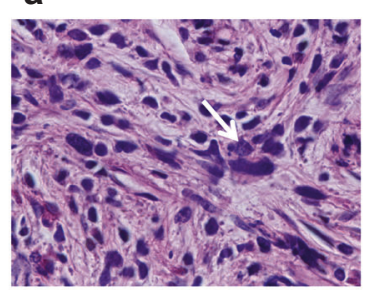

C

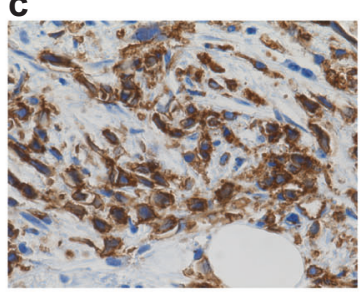

b

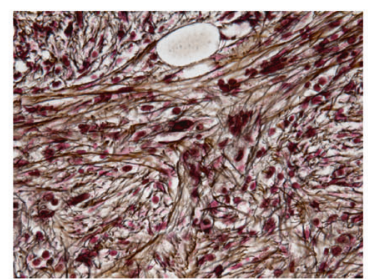

d

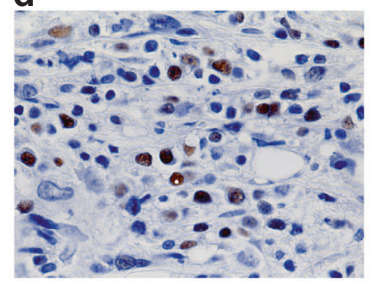

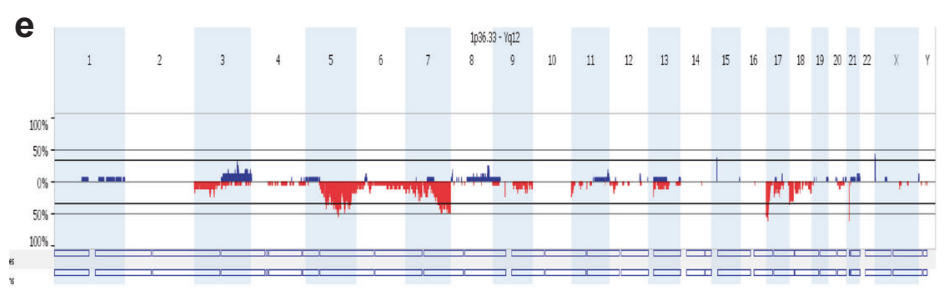

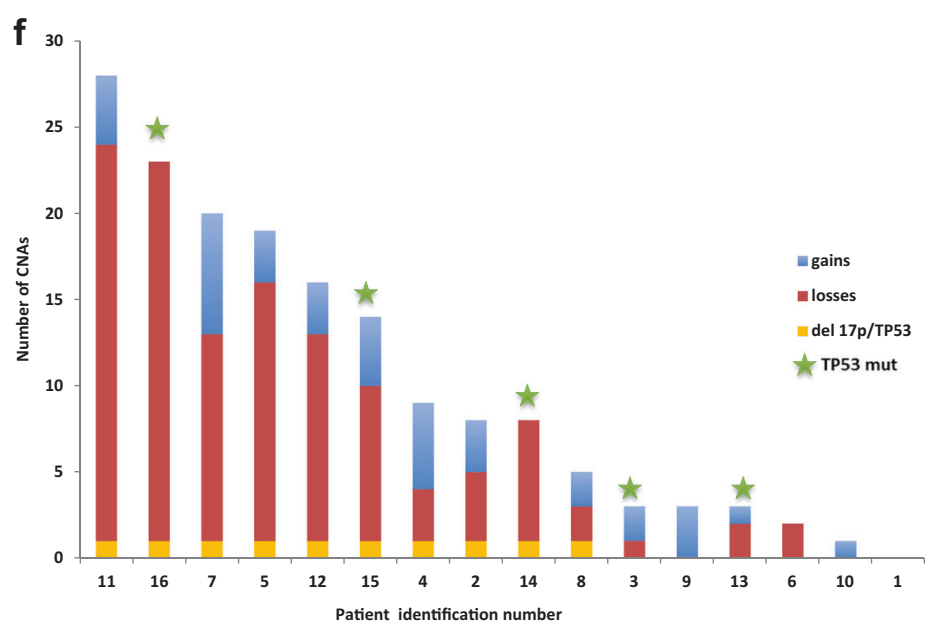

g

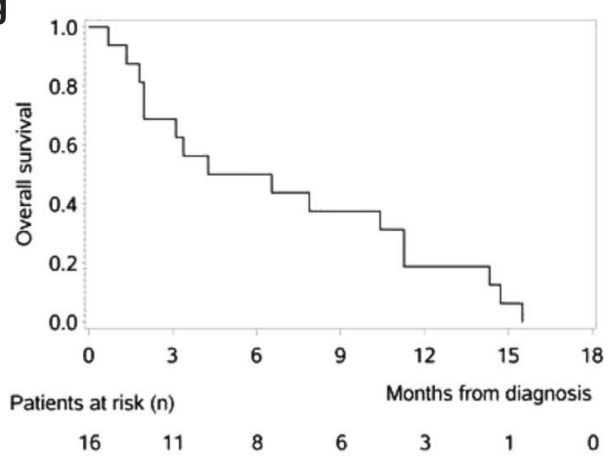

h
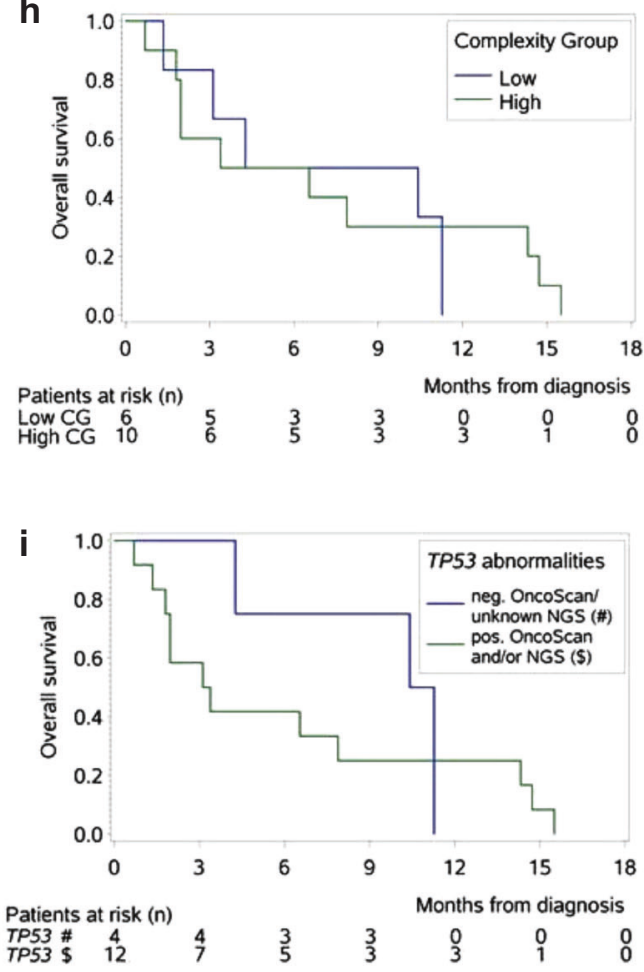

Fig. 1 Representative BM trephine biopsy, summary of the OncoScan copy number analysis and Kaplan-Meier analysis of overall survival. a-d BM morphology in a patient with high complexity genetic aberrations: a BM spaces are nearly devoid of fat cells and infiltrated by blast cells and residual dysplastic hematopoietic cells including micromegakaryocytes (arrow, hematoxylin and eosin, x63). b Diffuse densely increased reticulin fiber meshwork consistent with BM fibrosis grade 3 (Gomori's reticulin stain, $\times 63$ ). c Accumulation of CD34positive blast cells (CD34 immunohistochemistry, $\times 63)$. d Nuclear overexpression of the $\mathrm{p} 53$ protein in about $30 \%$ of nucleated cells (p53 immunohistochemistry, $\times 100)$. e Summary of the OncoScan copy

as well as trisomy 8 were considered a distinct category [12]. Patients in this subgroup were older and had the worst overall survival of all AML subgroups [11]. Accordingly, the term "AML with TP53 mutations and chromosomal aneuploidy" could also be assigned to most of our APMF patients. The number analysis showing the type of CNAs per chromosome in all patients using Nexus Express Software for OncoScan, blue color presents copy number gains and red color copy number losses. f Summary of the OncoScan copy number analysis showing the number of CNAs per patient. Stars inidicate the TP53 mutations in five patients identified via panel sequencing. g Kaplan-Meier analysis of overall survival (OS) of the total cohort. h Kaplan-Meier analysis of OS according to genomic complexity group. i Kaplan-Meier analysis of OS according to TP53 status as defined by: (\#) no TP53 aberrations in the OncoScan assay and unknown mutational status by NGS; (\$) TP53 aberrations in the OncoScan assay and/or TP53 mutations by NGS

detection of a JAK2 V617F mutation in one patient does not exclude de novo AML but may suggest a rapidly transformed MPN without a clinically evident chronic phase [13, 14]. MPN patients with TP53 mutations have a poor prognosis with a high risk of transformation [14]. Very high risk PMF 


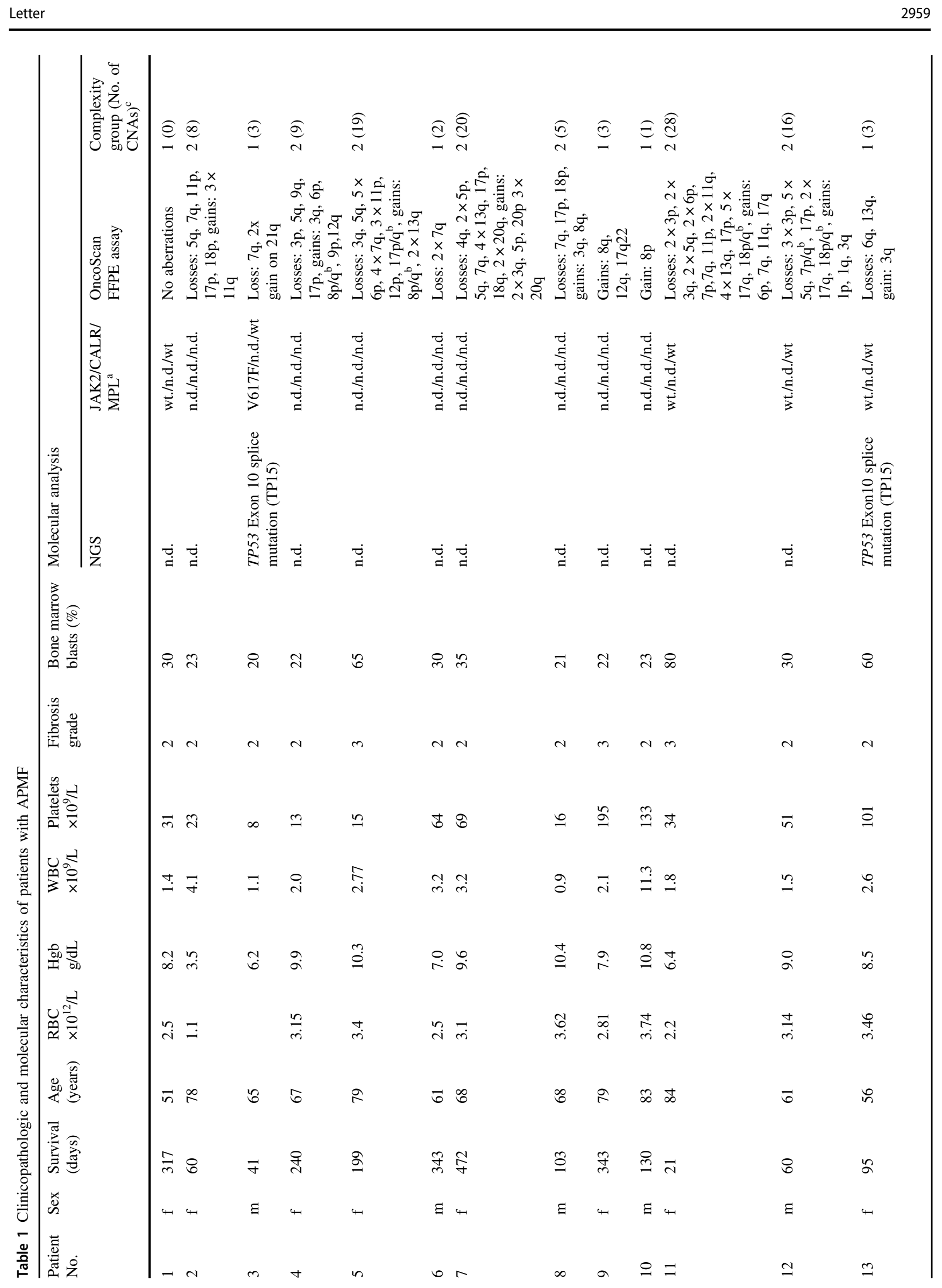

SPRINGER NATURE 


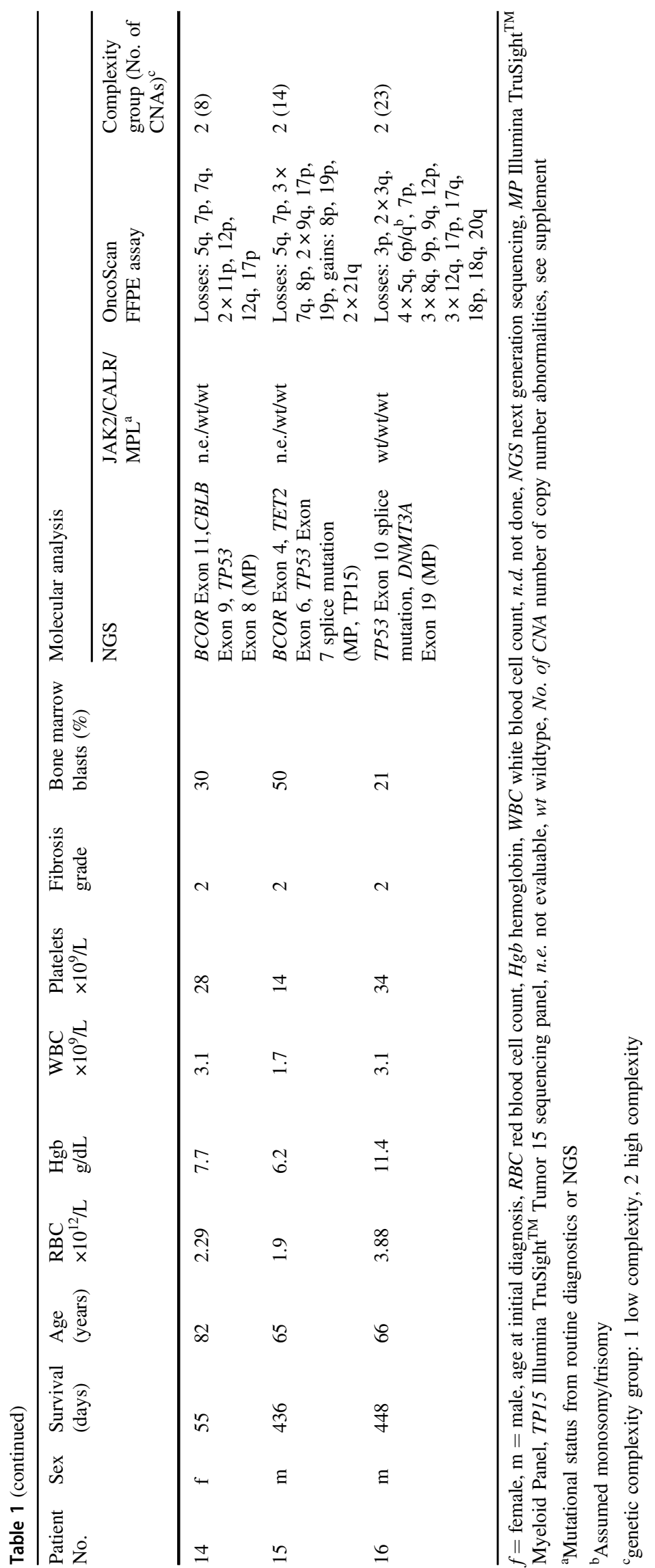


overlap at least in loss of $17 \mathrm{p}$ with APMF but the most frequent CNAs reported in PMF affect different chromosomal regions: 20q, 17q, 7p, 9p, 13q, or 1q [15]. Therefore, our results could be helpful in distinguishing APMF from PMF.

Despite the molecular heterogeneity, all patients of our APMF cohort had a dismal outcome with a median survival of 5.4 months (range 1.8-11.3 months, Fig. 1g-i, Supplementary Table 1). No patient was treated by allogeneic hematopoietic stem cell transplantation. Three patients received standard induction chemotherapy, one patient thalidomide and another patient decitabine. Statistical analyses comparing low versus high complexity cases or those with known or unknown TP53 abnormalities did not reveal any significant differences with regard to overall survival and hematologic pretreatment parameters, except with regard to platelet counts and number of CNAs (Fig. 1, Supplementary Table 1). Patients negative for TP53 abnormalities in the OncoScan assay and unknown TP53 mutation status had significantly higher platelet counts $(p=0.045)$ and lower numbers of CNAs $(p=0.006)$ than those with TP53 abnormalities. It is worth noting that the superior survival of 11.3 months was observed in a patient of the high complexity group with a TP53 exon 10 splice site mutation receiving hypomethylating therapy (decitabine). Interestingly, the frequency of TP53 splice mutations $(4 / 5,80 \%)$ in our cohort is high, compared to the splice mutation frequency of around $5 \%$ in myeloid neoplasms (11/201, see Suppl.) estimated from the IARC TP53 database. Whether this observation can be confirmed and may even be linked to the fibrotic phenotype needs to be shown in a larger cohort.

The mechanisms that contribute to the typical morphologic and clinical features, and the rapidly progressive disease that entailed the designation as APMF still remain to be elucidated. Previously, we have observed an increased inflammatory T-cell rich background in APMF core biopsies [5]. Inflammatory microenvironment changes of the niche may contribute to genomic instability of hematopoietic stem cells and disease evolution. Cytokines and chemokines released from inflammatory cells and abundant megakaryocytes may contribute to the fibrotic modulation of the BM that is a hallmark of APMF. Although the number of our patients is small and the spectrum of methods that we could apply is limited, the data presented here provide further insights into the molecular basis of APMF and highlight the high prevalence of TP53 abnormalities and chromosomal aneuploidy.

Author contributions All authors have reviewed and approved the manuscript. MP and DP designed the study, performed the OncoScan FFPE assay, the mutational analysis, the interpretation of results and contributed to manuscript writing. AS-G designed the study, performed the histopathological evaluation, and wrote the initial draft of the manuscript. SK-S retrieved the cohort, collected clinicopathologic, and molecular data, performed immunohistochemical stains and contributed to the histopathologic evaluation. GI performed the statistical analysis. HB, RZ, and JD contributed with analysis and interpretation of data, reviewed and approved the manuscript.

\section{Compliance with ethical standards}

Conflict of interest The authors declare that they have no conflict of interest.

Publisher's note: Springer Nature remains neutral with regard to jurisdictional claims in published maps and institutional affiliations.

Open Access This article is licensed under a Creative Commons Attribution 4.0 International License, which permits use, sharing, adaptation, distribution and reproduction in any medium or format, as long as you give appropriate credit to the original author(s) and the source, provide a link to the Creative Commons license, and indicate if changes were made. The images or other third party material in this article are included in the article's Creative Commons license, unless indicated otherwise in a credit line to the material. If material is not included in the article's Creative Commons license and your intended use is not permitted by statutory regulation or exceeds the permitted use, you will need to obtain permission directly from the copyright holder. To view a copy of this license, visit http://creativecommons. org/licenses/by/4.0/.

\section{References}

1. Arber DA, Orazi A, Hasserjian R, Thiele J, Borowitz MJ, Le Beau MM, et al. The 2016 revision to the World Health Organization classification of myeloid neoplasms and acute leukemia. Blood. 2016;127:2391-405.

2. Arber DA, Brunning RD, Orazi A, Porwit A. Acute myeloid leukemia, NOS. In: Swerdlow SH, Campo E, Harris NL, et al., editors. WHO classification of tumours of haematopoietic and lymphoid tissues. 4th ed. Lyon, France: International Agency for Research on Cancer; 2017.

3. Orazi A, O’Malley DP, Jiang J, Vance GH, Thomas J, Czader M, et al. Acute panmyelosis with myelofibrosis: an entity distinct from acute megakaryoblastic leukemia. Mod Pathol. 2005;18:603-14.

4. $\mathrm{Xu}$ Zhaodong. AML with myelodysplasia-related changes masquerades as acute panmyelosis with myelofibrosis. Blood. 2017; 130:1775.

5. Thiele J, Kvasnicka HM, Schmitt-Graeff A. Acute panmyelosis with myelofibrosis. Leuk Lymphoma. 2004;45:681-7.

6. Suvajdzic N, Marisavljevic D, Kraguljac N, Pantic M, Djordjevic V, Jankovic G, et al. Acute panmyelosis with myelofibrosis: clinical, immunophenotypic and cytogenetic study of twelve cases. Leuk Lymphoma. 2004;45:1873-9.

7. Bae E, Park CJ, Cho YU, Seo EJ, Chi HS, Jang S, et al. Differential diagnosis of myelofibrosis based on WHO 2008 criteria: acute panmyelosis with myelofibrosis, acute megakaryoblastic leukemia with myelofibrosis, primary myelofibrosis and myelodysplastic syndrome with myelofibrosis. Int $\mathrm{J}$ Lab Hematol. 2013;35:629-36.

8. Grygalewicz B, Woroniecka R, Pastwińska A, Rygier J, Krawczyk P, Borg K, et al. Acute panmyelosis with myelofibrosis with EVI1 amplification. Cancer Genet. 2012;205:255-60.

9. Kim SY, Im K, Park SN, et al. CALR, JAK2, and MPL mutation profiles in patients with four different subtypes of myeloproliferative neoplasms: primary myelofibrosis, essentialthrombocythemia, polycythemia vera, and myeloproliferative neopl neoplasm, unclassifiable. Am J Clin Pathol 2015;143:635-44. 
10. Foster JM, Oumie A, Togneri FS, Vasques FR, Hau D, Taylor M, et al. Cross-laboratory validation of the OncoScan FFPE Assay, a multiplex tool for whole genome tumour profiling. BMC Med Genom. 2015;8:5.

11. Rücker FG, Schlenk RF, Bullinger L, Kayser S, Teleanu V, Kett H, et al. TP53 alterations in acute myeloid leukemia with complex karyotype correlate with specific copy number alterations, monosomal karyotype, and dismal outcome. Blood. 2012;119:2114-21.

12. Papaemmanuil E, Gerstung M, Bullinger L, Gaidzik VI, Paschka $\mathrm{P}$, Roberts ND, et al. Genomic classification and prognosis in acute myeloid leukemia. N Engl J Med. 2016;374:2209-21.
13. Aynardi J, Manur R, Hess PR, Chekol S, Morrissette JJD, Babushok D, et al. JAK2 V617F-positive acute myeloid leukaemia (AML): a comparison between de novo AML and secondary AML transformed from an underlying myeloproliferative neoplasm. A study from the Bone Marrow Pathology Group. Br J Haematol. 2018;182:78-85.

14. Grinfeld J, Nangalia J, Baxter EJ, Wedge DC, Angelopoulos N, Cantrill $\mathrm{R}$, et al. Classification and personalized prognosis in myeloproliferative neoplasms. N Engl J Med. 2018;379:1416-30.

15. Tefferi A, Guglielmelli P, Nicolosi M, Mannelli F, Mudireddy M, Bartalucci N, et al. GIPSS: genetically inspired prognostic scoring system for primary myelofibrosis. Leukemia. 2018;32:1631-42.

Leukemia (2019) 33:2962-2966

https://doi.org/10.1038/s41375-019-0528-3

Acute myeloid leukemia

\title{
Phorbol ester induced ex vivo expansion of rigorously-defined phenotypic but not functional human cord blood hematopoietic stem cells: a cautionary tale demonstrating that phenotype does not always recapitulate stem cell function
}

\author{
Yandan Chen ${ }^{1} \cdot$ Chunxu Yao ${ }^{1} \cdot$ Yincheng Teng $^{2} \cdot$ Rongzhen Jiang $^{2} \cdot$ Xinxin Huang ${ }^{3} \cdot$ Sheng Liu ${ }^{4}$ Jun Wan ${ }^{4,5}$. \\ Hal E. Broxmeyer ${ }^{3} \cdot$ Bin Guo $^{1}$
}

Received: 17 April 2019 / Revised: 16 May 2019 / Accepted: 22 May 2019 / Published online: 26 July 2019

(c) Springer Nature Limited 2019

These authors contributed equally: Yandan Chen, Chunxu Yao

Supplementary information The online version of this article (https:// doi.org/10.1038/s41375-019-0528-3) contains supplementary material, which is available to authorized users.

Hal E. Broxmeyer

hbroxmey@iupui.edu

$\triangle$ Bin Guo

jybinguo@shsmu.edu.cn

1 Department of Pathophysiology, Key Laboratory of Cell Differentiation and Apoptosis of Chinese Ministry of Education, Shanghai Jiao Tong University School of Medicine, Shanghai 200025, China

2 Obstetric Intensive Care Center, The Institute of Obstetrics and Gynecology, Department of Obstetrics and Gynecology, Affiliated Sixth People's Hospital, Shanghai Jiao Tong University, Shanghai 200233, China

3 Department of Microbiology and Immunology, Indiana University School of Medicine, Indianapolis, IN 46202, USA

4 Department of Medical and Molecular Genetics, Center for Computational Biology and Bioinformatics (CCBB), Indiana University School of Medicine, Indianapolis, IN 46202, USA

5 Department of BioHealth Informatics, School of Informatics and Computing, Indiana University Purdue University Indianapolis (IUPUI), Indianapolis, IN 46202, USA

\section{To the Editor:}

Hematopoietic stem cells (HSCs) are a rare population residing at the apex of the hematopoietic hierarchy [1]. HSCs have the capacity to self-renew and differentiate into all blood cell types, thus they play a key role in hematopoietic cell transplantation (HCT) [2]. HCT is widely used as a curative therapy for numerous malignant and nonmalignant hematological and even non-hematological diseases [3]. The fast developing field of gene editing techniques, including ZFNs, TALENs and CRISPR-Cas9, broaden usage of HCT in clinical therapy of diseases caused by genetic mutations [4]. i.e. $\beta$-thalassaemia or Sickle Cell Disease (SCD) may possibly be interrogated by CRISPR based gene editing of $\beta$-globin in HSCs from patients [5]. However, efficient gene editing and infusion of the gene edited HSC into patients requires sufficient numbers of donor HSC. In addition, when cord blood (CB) is used as the source of HSC for HCT, rare HSC numbers in single CB units may lead to delayed hematopoietic recovery in recipients [6]. It is thus important in some cases to develop efficient means that can overcome limited HSC numbers to enhance the efficacy of HCT. 